\title{
Effects of gaze angle and extraretinal eye movement information on visual locomotion control
}

\author{
JEREMY M. A. BEER \\ Veridian, Inc., Brooks Air Force Base, Texas
}

\begin{abstract}
This study tested effects of gaze-movement angle and extraretinal eye movement information on performance in a locomotion control task. Subjects hovered in a virtual scene to maintain position against optically simulated gusts. Gaze angle was manipulated by varying the simulated camera pitch orientation. Availability of extraretinal information was manipulated via simulated-pursuit fixation. In Experiment 1 , subjects performed better when the camera faced a location on the ground than when it pointed toward the horizon. Experiment 2 tested whether this gain was influenced by availability of appropriate eye movements. Subjects performed slightly better when the camera pointed at nearby than at distant terrain, both in displays that did and in displays that did not simulate pursuit fixation. This suggested that subjects could perform the task using geometric image transformations, with or without appropriate eye movements. Experiment 3 tested more rigorously the relative importance of gaze angle and extraretinal information over a greater range of camera orientations; although subjects could use image transformations alone to control position adequately with a distant point of regard, they required eye movements for optimal performance when viewing nearby terrain.
\end{abstract}

As an observer moves through a visual scene, he/she typically fixates a succession of stationary objects in that scene. When observers are required to control locomotion visually, therefore, they must extract meaningful information about their own movement during these gaze fixations. ${ }^{1}$ Two factors that merit evaluation as determinants of locomotion control performance are gazemovement angle and the availability of extraretinal eye movement information. This is because both factors have been shown to influence self-motion perception under passive viewing conditions (Banks, Ehrlich, Backus, \& Crowell, 1996; Beusmans, 1994; Cutting, 1986; Cutting, Springer, Braren, \& Johnson, 1992; Cutting, Vishton, Flückiger, Baumberger, \& Gerndt, 1997; Ehrlich, Beck, Crowell, Freeman, \& Banks, 1998; Royden, 1994; Royden, Banks, \& Crowell, 1992; Royden, Crowell, \& Banks, 1994; Vishton \& Cutting, 1995; W. H. Warren \& Hannon, 1988, 1990; W. H. Warren, Morris, \& Kalish, 1988), and both can vary widely as the moving viewer shifts gaze from one location in the scene to another (or progresses

This research was conducted under a National Research Council Research Associateship at the U.S. Air Force Armstrong Laboratory, Wright-Patterson Air Force Base, Ohio, and continued under the support of Grant 2313 W702 from the U.S. Air Force Office of Scientific Research to the Armstrong Laboratory Crew Technology Division, Brooks Air Force Base, Texas. I am grateful for the contributions of Fred Previc, Rik Warren, and Sheila Garness. The views expressed in this paper are those of the author and do not necessarily reflect the views of the U.S. Air Force, the Department of Defense, or Veridian, Inc. Correspondence should be addressed to J. M. A. Beer, Veridian, Inc., PO Box 35482, Brooks AFB, TX 78235-5104 (e-mail: jbeer@) satx.veridian,com). through the scene while maintaining fixation on a single location). Since perceptual performance can be influenced by the mobile observer's actions and vice versa (Gibson, 1979; W. H. Warren, 1990), it is desirable to determine whether gaze-movement angle and extraretinal eye movement information influence active locomotion control as they have been shown to influence passive selfmotion perception. This paper describes three experiments performed to examine these questions. In Experiments $1-3$, gaze-movement angle was found to affect moving subjects' ability to respond to simulated gust disturbances while hovering over a flat plane. In Experiment 3, this effect was found to interact with the availability of extraretinal information.

\section{Gaze-Movement Angle}

Gaze-movement angle is the angle formed between the moving viewer's direction of gaze and that viewer's instantaneous direction of movement (Cutting, 1986). Gazemovement angle is an important factor influencing selfmotion perception because the geometric structure and magnitude of the available visual information vary widely according to where the viewer looks in the environment; this is true even for subjects moving in uncluttered environments, and even before the complicating effects of eye rotation are considered (Koenderink, 1986; Stoffregen, 1985; W. H. Warren et al., 1988). An example of how the geometric structure of visual information can change with gaze direction is the distinction between the radial expansion pattern seen by a moving observer looking toward the heading aim point and the parallel (lamellar) flow pattern seen when the observer looks in a direction per- 
pendicular to the heading. An example of how the magnitude of the visual information can change with gaze direction is the difference between the barely perceptible optical expansion observed by an organism looking toward the heading aim point on the horizon while moving over a flat terrain, and the more dramatic expansion that can be seen when the creature continues to look forward but also downward, at nearby terrain below the horizon (Gibson, 1950; W. H. Warren et al., 1988).

Several studies have used displays depicting passive self-motion to examine the effects of manipulating gazemovement angle on various aspects of heading perception. ${ }^{2}$ Increasing the gaze-movement angle improves relative discrimination between gaze and heading directions (Cutting et al., 1997); when subjects were required to judge whether they were heading to the left or the right of a fixated target in simulations of linear self-motion, responses were more accurate at greater gaze-movement angles (Cutting, 1986; Cutting et al., 1992; Cutting et al., 1997; W. H. Warren \& Hannon, 1990; W. H. Warren et al., 1988). In contrast, studies examining subjects' directional estimates of absolute heading in passive self-motion simulations showed that judgments were more accurate when smaller eccentricities (i.e., gaze-movement angles) separated gaze direction and heading (Crowell \& Banks, 1993; Cutting et al., 1997; R. Warren, 1976; W. H. Warren \& Kurtz, 1992).

The effects of gaze-movement angle on performance in both of these passive judgment tasks (namely, directional gaze-heading discrimination and absolute heading estimation) are potentially important for active locomotion control, though not necessarily in equivalent ways. One of the most important tasks in locomotion is the perception of heading relative to looming obstacles or landmarks (Cutting et al., 1992; Cutting et al., 1997). To the extent that the observer usually fixates these looming objects as he/she progresses, discriminating between gaze and heading directions can be important for locomotion control. Increasing the gaze-movement angle should therefore help performance in certain locomotion tasks because it facilitates gaze-heading discriminations. If, for example, a hovering observer is attempting to maintain position over a fixed location on the ground while being buffeted in the wind, he/she will likely fixate that location while trying to correct for each gust. In this situation, it is more important to be able to detect gusts rapidly and make gross discriminations between forward and backward, upward and downward, or leftward and rightward gusts (which in certain cases can be accomplished through simple gaze-heading discrimination) than it is to point exactly toward the heading aim point.

Other locomotion tasks such as vehicle steering depend on heading perception in the absolute reference frame in addition to gaze-heading discrimination. While it is important for the driver to determine whether the vehicle will pass to the left or right of an obstacle looming ahead, it is just as important for the driver to maintain continu- ous awareness of the vehicle's absolute heading. The latter task is best served by keeping the gaze-movement angle small. A practical manifestation of this principle is the well-known "look where you want to go" rule, according to which drivers should look as far ahead on the road as possible (Bondurant \& Blakemore, 1982, p. 107). To illustrate the difficulty of extracting absolute heading information while fixating objects at wide and rapidly increasing gaze-movement angles, one can imagine driving a sports car while looking at telephone poles through the side window.

Since locomotion control depends on accurate selfmotion perception, and gaze-movement angle has been shown to affect the accuracy of self-motion perception, it is reasonable to test empirically the extent to which gazemovement angle influences locomotion control performance.

\section{Extraretinal Eye Movement Information}

When a moving eye fixates a stationary object in the scene, the resulting ocular pursuit is analogous to a cinematic combination of dolly and pan (Cutting et al., 1992; Cutting et al., 1997; Hochberg, 1986, Figure 22.14) in which the moving camera rotates to keep the fixated landmark at the center of the screen. Ocular pursuit complicates the problem of using the flow field to determine heading because it adds a rotational flow component, but in spite of this, moving subjects can usually judge heading accurately during ocular pursuit (Banks et al., 1996; Regan \& Beverley, 1982; Royden et al., 1992; Royden et al., 1994; W. H. Warren \& Hannon, 1988, 1990).

Extraretinal models of heading perception account for this by using eye movement information from the oculomotor system to determine the rotational component and then subtracting this from the flow field to obtain the translation solution (Royden et al., 1994; von Holst, 1954; see also Wertheim, 1990, p. 173). Retinal image models, in contrast, derive a heading solution using only the visual information projected on the retina (Hildreth, 1992; Longuet-Higgins \& Prazdny, 1980; Perrone, 1992; Rieger \& Lawton, 1985). A number of studies have been performed to distinguish the two models by comparing absolute heading estimates between (1) conditions in which observers actually moved their eyes and (2) conditions involving simulated pursuit, in which observers' eyes remained stationary and the display simulated the visual effects of eye rotation. Whereas retinal image models predict heading estimates of equal accuracy in real- and simulated-pursuit conditions, extraretinal models predict that estimates will be more accurate in real-pursuit conditions and will suffer systematic directional errors in simulated-pursuit conditions. In general, more accurate heading estimates have been obtained in real-pursuit than in simulated-pursuit conditions when rotational pursuit speeds were greater than $1 \% / \mathrm{sec}$ (which, as Royden et al. [1992] pointed out, is generally the case for observers moving in the real world); this indicates that ex- 
traretinal information is necessary for accurate heading perception (Banks et al. 1996; Beusmans, 1994; Royden et al., 1992; Royden et al., 1994; W. H. Warren \& Hannon, 1988, 1990).

When viewing simulated-pursuit displays, observers typically commit systematic estimation errors in the direction of the simulated rotation (Royden, 1994; Royden et al., 1992; Royden et al., 1994). These errors are often accompanied by a misperception of curvilinear translation veering in the direction of error. Banks et al. (1996) determined that the extent of this estimation error in simulated-pursuit displays is proportional to the amount of simulated rotation. An explanation for this is that following subtraction of the oculomotor pursuit component, the visual system attributes any rotation remaining in the retinal flow field to a change in heading (Banks et al., 1996; Royden, 1994; W. H. Warren, Mestre, Blackwell, \& Morris, 1991). ${ }^{3}$

Considering the above findings in conjunction with the perceptual demands associated with locomotion makes it reasonable to expect extraretinal eye movement information to influence not only passive self-motion perception but also self-motion control. One way to test this is to measure performance in locomotion control tasks, such as position maintenance and steering, in real-versus simulatedpursuit conditions. Since extraretinal information has been shown to be necessary for accurate interpretation of the retinal flow field, errors such as the curved-path misperception could in some situations cause observers to make inappropriate control inputs when visual pursuit is simulated by the display and not executed by the oculomotor system.

\section{The Hover Task}

Because locomotion is an ongoing control process, the experiments described in this paper were intended to measure control performance throughout extended trials as opposed to short (only 1- or 2-sec) depictions of selfmotion. For this reason, a locomotion task was selected that would accommodate the manipulation of gazemovement angle and extraretinal eye movement information while also allowing these factors to remain relatively constant throughout each trial. This is difficult to accomplish in a depiction of continuous forward movement because if the viewer or camera fixates one location in the scene, both the gaze-movement angle and the required ocular pursuit speed can change dramatically throughout the movement. Instead of continuous forward flight, the experiments used a hover task in which the viewer maintained position while being buffeted by optically simulated wind gusts and while fixating a location on the horizon or on the ground ahead. This approach resembles that used in an existing body of research examining subjects' ability to regulate altitude in scenes with various types of ground texture (Flach, Hagen, \& Larish, 1992; Garness, Flach, Stanard, \& Warren, 1994; Johnson, Tsang, Bennett, \& Phatak, 1989; R. Warren, 1988); the present experiments, however, required subjects to regulate fore-aft position, not altitude. Since hover requires the observer to remain in approximately the same location, this allowed the maintenance of a relatively uniform gaze-movement angle throughout each trial while still allowing the depiction of viewpoint displacements via the simulated wind gusts.

In this task, subjects used a joystick to correct for strong gusts that pushed them forward or backward; the stick allowed them to regulate position along the forwardbackward dimension only. Weaker vertical (up-down) gusts were also included, which required subjects to distinguish image transformations associated with forwardbackward translation from transformations associated with changing altitude; this reduced the possibility of subjects' adopting inappropriate control strategies (Flach et al., 1992). Gaze-movement angle was manipulated in these experiments by varying the pitch orientation of the virtual camera, ${ }^{4}$ while the availability of extraretinal eye movement information was manipulated via the inclusion or omission of simulated-pursuit fixation.

\section{EXPERIMENT 1 Self-Motion Control With Simulated-Pursuit Fixation Displays}

Experiment 1 was conducted to determine whether observers could perform the position control task in simulated-pursuit conditions. The camera's initial pitch orientation (its pitch angle at the beginning of each trial) was varied across three conditions, ranging from a baseline value of zero (pointing continuously straight out to the horizon) to $-45^{\circ}$ (simulating pursuit of a ground location 1 eye-height ahead on the ground). This manipulation was tantamount to presenting three different simulatedpursuit conditions among which only camera orientation was varied; in the baseline configuration, however, the camera was effectively pursuing an infinitely distant point, so no pursuit rotation was required and the simulated gaze-movement angle remained near zero (for forward gusts) or $180^{\circ}$ (for backward gusts). ${ }^{5}$ The baseline condition was called the "horizon condition."

The remaining two conditions included simulated fixation whereby the camera pursued a fixed location on the ground ahead; as the camera moved forward and back, it would pan down and up, respectively, to keep this location centered on the screen. The camera's initial pitch angle, and hence the simulated gaze-movement angle associated with the forward-back gusts, were varied between the two simulated-pursuit conditions. In the $-18^{\circ}$ condition, the camera began each trial fixating a distant ground location. In the $-45^{\circ}$ condition, the camera began each trial fixating a location only 1 eye-height ahead on the ground, and the gaze-movement angle associated with the forwardback gusts was thus much greater.

Indices of manual control performance were measured across each trial to determine the effectiveness with which 

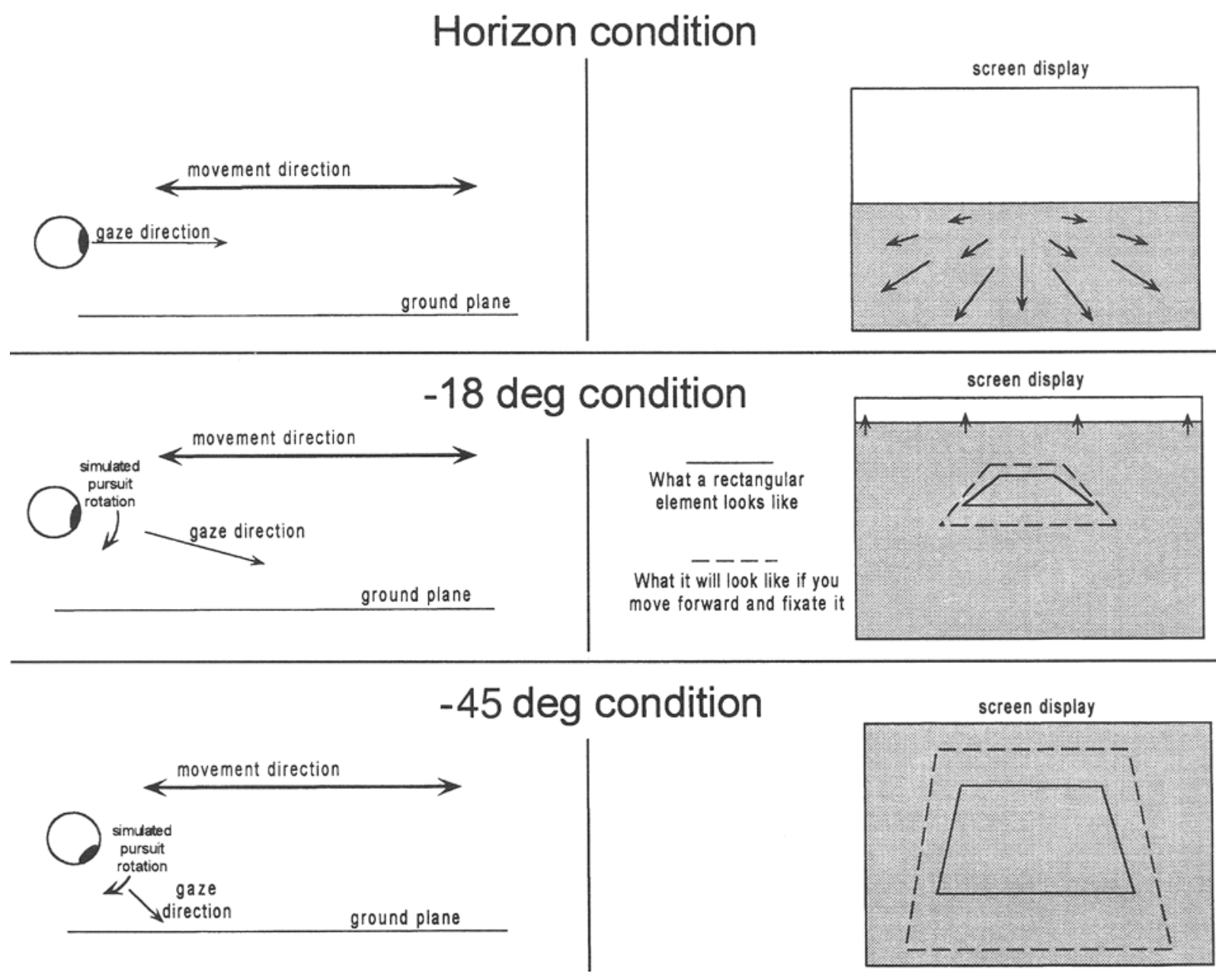

Figure 1. Layout for horizon (top), $-18^{\circ}$ (middle), and $-45^{\circ}$ (bottom) conditions, Experiment 1. Note that in $-18^{\circ}$ displays, forward motion causes the horizon to rise on the screen. (Figure is not to scale. Left panels show side views of the layout of the virtual eye point and the ground. Right panels represent the screen displays for these conditions, including the image transformations that would result from depicting forward movement.)

subjects could detect each gust displacement, determine its direction, and execute the appropriate correction with the joystick. In the event of a vertical gust, this correction would be a null response. Since the gusts were predominantly in the forward-backward dimension, however, subjects could perform the task efficiently through gaze-heading discrimination in the two simulatedpursuit conditions. ${ }^{6}$ If the heading lay above the gaze direction, this would indicate forward displacement, whereas a backward gust could be recognized by registering a heading below the gaze direction (Figure 1). Since gazeheading discrimination improves with increasing gazemovement angle in both real- and simulated-pursuit conditions (Cutting, 1986; Cutting et al., 1992; Cutting et al., 1997; W. H. Warren \& Hannon, 1990; W. H. Warren et al., 1988), it was expected that the two simulatedpursuit conditions would yield better position control performance than the horizon condition, and that perfor- mance in the $-45^{\circ}$ condition would surpass performance in the $-18^{\circ}$ condition.

\section{Method}

Subjects. Six male and 6 female Wright State University undergraduates with normal or corrected vision were paid to participate. The experiment was completed in two 90 -min sessions.

Stimuli. Trials were 100 -sec computer generated $1024 \times 768$ pixel real-time animation sequences. The frame rate was $15.5 / \mathrm{sec}$. The display screen was $1.7 \mathrm{~m}$ high and $2.3 \mathrm{~m}$ wide. Viewing position was at the projection center, $1.8 \mathrm{~m}$ from the screen, yielding an image $52^{\circ}$ high and $66^{\circ}$ wide.

The ground was indicated by a rectangular island of checkered light and dark-green squares. The ground was light green outside the island. A blue sky was displayed above the horizon. Projective dimensions were consistent with a scene layout in which the island was $49.4 \mathrm{~m}$ wide and $73.2 \mathrm{~m}$ long. The island was divided into 27 columns and 40 rows; each square was $1.83 \mathrm{~m}$ on a side. The camera began each trial at an altitude of $4.57 \mathrm{~m}$ directly above a point $7.6 \mathrm{~m}$ from one end of the island, and looked toward the other end. 
Gusts were simulated by applying velocity disturbances to the camera along the forward-backward and altitude axes. Altitude gusts were included to prevent subjects from performing the task by merely aligning texture with the screen's edges. Time histories of gusts in each dimension were determined by superimposing five sine waves of differing frequency and amplitude. Forward-backward and altitude velocities varied over time according to the resulting waveforms; this made the gusts appear to vary randomly in direction and strength. No component frequency was an integral multiple of any other in either gust history. Forward-backward component frequencies were $11,41,71,89$, and 113 cycles per 100 -sec data interval; components cycled between adding forward or backward velocity at $.11, .41, .71, .89$, and $1.13 \mathrm{~Hz}$, respectively. Following an assumption that low-frequency natural disturbances are strongest, peak speed was $\pm 1.34 \mathrm{~m} / \mathrm{sec}$ for the two lowest frequency components and $\pm 0.60 \mathrm{~m} / \mathrm{sec}$ for the remaining components. Frequencies for the altitude velocity components were 13 , $29,47,79$, and 103 cycles per trial, equivalent to $13, .29, .47, .79$, and $1.03 \mathrm{~Hz}$, respectively. Peak speeds were $0.80 \mathrm{~m} / \mathrm{sec}$ for the two lowest frequency altitude components and $0.36 \mathrm{~m} / \mathrm{sec}$ for the remaining components. With peak speed $A$ and frequency $F$, each vertical velocity component would cause an altitude excursion of $A /(\pi * F)$ if imposed by itself. ${ }^{7}$ The camera was subjected to the same gust histories across all trials. The maximum and minimum altitudes reached were 4.67 and $1.48 \mathrm{~m}$. Forward and backward limitations of the camera's travel were dependent on the subject's performance in each trial.

The camera's orientation was manipulated between conditions. In the horizon condition, the camera pointed forward and the horizon was stationary on the screen throughout the trial. The island's far edge was visible continuously; its screen position moved up and down slightly, opposite the altitude gusts' direction. In the remaining two conditions, the display simulated fixation by panning the camera up and down over time to maintain at the screen's center the image of a fixed location on the ground. In the $-18^{\circ}$ condition, the camera pointed $18^{\circ}$ below the horizon at the start of each trial and pursued a fixation location that was initially $13.7 \mathrm{~m}$ (3.0 times the initial eye-height) in front of the eye point. The horizon and the island's far edge were usually in view in this condition. When forward or upward gusts occurred, the camera rotated downward, and the horizon and the island's far edge moved upward slightly on the screen; the opposite occurred when backward or downward gusts occurred. In the $-45^{\circ}$ condition, the camera began each trial pitched $45^{\circ}$ downward from the horizon; the fixation location was initially $4.57 \mathrm{~m}$ (1.0 times initial eye-height) ahead. Angular speed of the pan rotations was higher in the $-45^{\circ}$ displays, which caused greater changes in the ground's slant relative to the gaze direction. Because of the steep pitch angle, the horizon and the island's far edge were usually not visible in this condition.

Design. Position error, or the distance in the forward-backward dimension between the instantaneous position and the starting position the subject was to maintain, was measured throughout each trial. Performance was assessed by calculating the mean, the absolute value of the mean, and the standard deviation of the position error over the trial. The signed mean error indicated any tendency for subjects to drift in a consistent direction throughout the trials. The absolute value of the mean error registered the extent to which subjects were drifting in either direction throughout the trials. The standard deviation of the position error indicated how much subjects were allowing gusts to move them back and forth; low values for this measure would indicate that the subject was correcting for the gusts rapidly and accurately.

The first session included presentation of all three conditions in one of six possible orders; the second session presented the same conditions in reverse order. Order was counterbalanced across subjects. Each of the six possible orders was presented to 2 subjects. Within a condition, subjects viewed the same display for six consecutive trials.
Apparatus. Displays were generated on a 33-MHz DOS computer incorporating XTAR Falcon 4000 animation and were projected on a front-projection screen by an Electrohome 38-B0988075 video projection system mounted above and behind the viewer. Motion was rendered by a simulation program (Engineering Solutions, Inc., Columbus, $\mathrm{OH}$ ) that generates virtual flight trajectories including sum-of-sines disturbance functions. The subject's position was recorded every fourth frame (every $0.258 \mathrm{sec}$ ). Subjects maintained position using a Gravis joystick; forward-backward deflections added a corresponding component to the eye point's speed on the forward-backward axis. This component varied with deflection up to a maximum of approximately $4.6 \mathrm{~m} / \mathrm{sec}$. This was sufficient to counter the strongest forward-backward gusts.

Procedure. Instructions described the island, gusts, and task. Subjects were informed that they could use the stick to control speed in the forward-backward dimension only. Instructions stated that the subject's objective was to stay positioned over the place on the ground that was under him/her at the start of the trial. Subjects were instructed to keep their gaze near the center of the screen and to try to respond to forward-backward gusts independent of altitude. It was explained that trying to keep a given feature stationary on the screen was not a good strategy. In the horizon condition, subjects were instructed that the screen would display a view through a camera pointing toward the horizon. In the $-18^{\circ}$ condition, subjects were informed that the camera would point at a location on the ground some distance ahead and would pivot up and down to pursue this location throughout the trials. In the $-45^{\circ}$ condition, subjects were instructed about the camera's pursuit of the nearby fixation location. Subjects pressed a button on the joystick to begin trials. In the first session, subjects completed five practice trials before each condition.

\section{Results and Discussion}

In each session, means were calculated across the six trials in each condition for the mean position error, the absolute value of mean position error, and the standard deviation of position error. Means were analyzed in a 3 (initial camera orientations) $\times 2$ (sessions) within-subjects analysis of variance. Data are summarized in Figure 2. There were main effects of initial camera orientation $[F(2,22)=29.1, p<.001]$ and session $[F(1,11)=10.7$, $p<.01]$ on mean position error. Pairwise Bonferroni comparisons (two-tailed $\alpha=.05$ ) identified as significant the difference between the horizon and the $-18^{\circ}$ conditions, but not the difference between the $-18^{\circ}$ and the $-45^{\circ}$ conditions. Interaction between initial camera orientation and session was not significant $[F(2,22)=3.0]$.

Similar effects of initial camera orientation $[F(2,22)=$ $14.9, p<.001]$ and session $[F(1,11)=7.8, p<.02]$ were observed for the absolute value of position error. Errors were highest in the horizon condition and were higher in the $-18^{\circ}$ than in the $-45^{\circ}$ condition. Bonferroni contrasts identified as significant the difference between the horizon and the $-18^{\circ}$ condition, but not between the $-18^{\circ}$ and the $-45^{\circ}$ condition. No significant interaction was observed $[F(2,22)=1.2]$.

There were main effects of initial camera orientation $[F(2,22)=17.4, p<.001]$ and session $[F(1,11)=15.7$, $p<.005]$ on the standard deviation of position error, with the highest values occurring in the horizon condition and higher values in the $-18^{\circ}$ than in the $-45^{\circ}$ condition. Bonferroni contrasts identified as significant the difference between the horizon and the $-18^{\circ}$ condition, but 


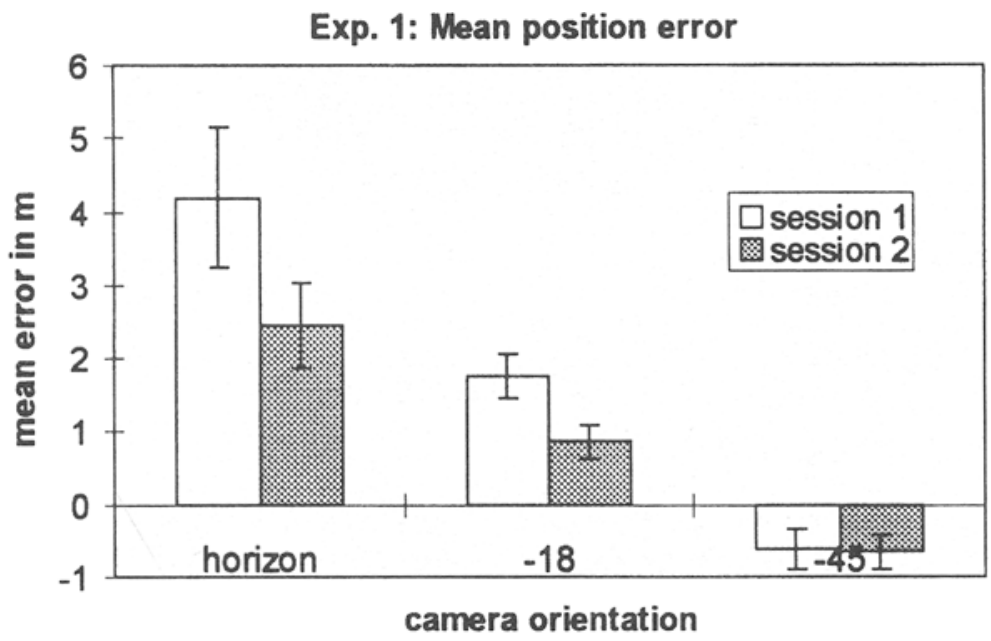

Exp. 1: Absolute value of mean position error

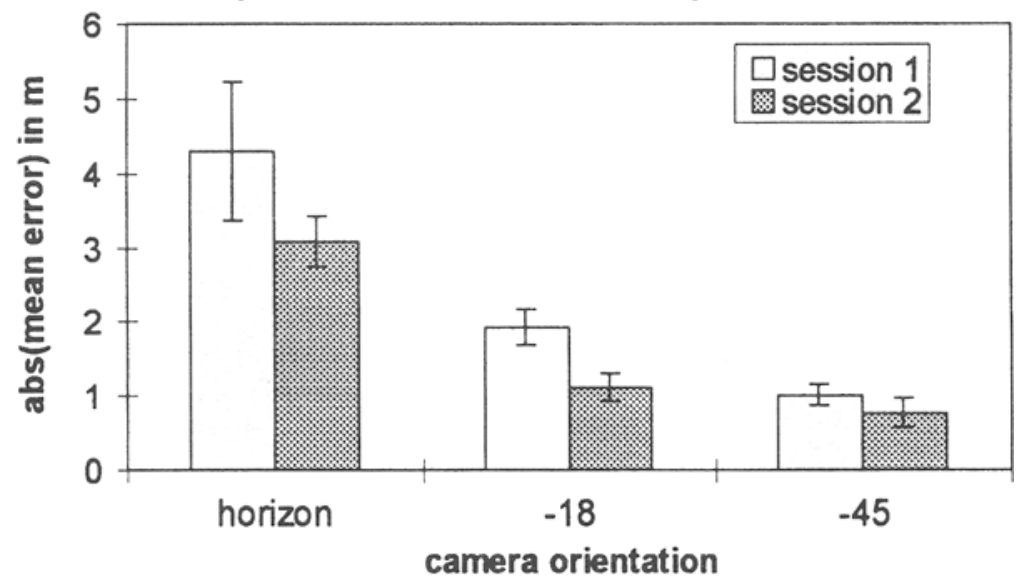

Exp. 1: Standard deviation of position

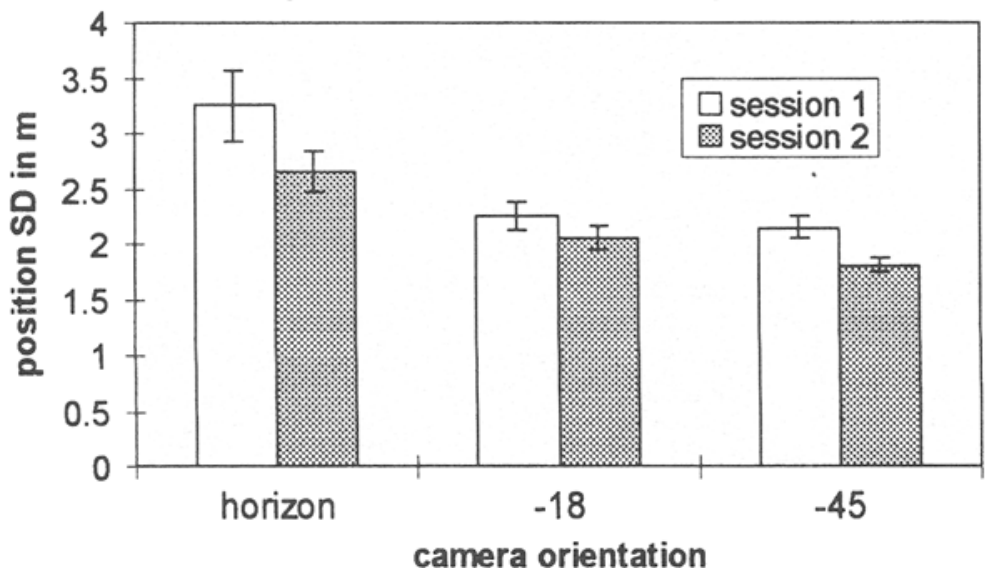

Figure 2. Upper panel: mean position error in Experiment 1. Middle panel: absolute values of mean position error, Experiment 1. Lower panel: standard deviations of position error, Experiment 1. (Values are means across subjects and repetitions, displayed by session and condition. Units are meters. Error bars represent $1 S E M$ for each condition.) 
not between the $-18^{\circ}$ and the $-45^{\circ}$ condition. There was no significant interaction $[F(2,22)=1.2]$.

The three performance measures indicated that position control was generally poorer in the horizon condition than in the other two conditions. The positive (forward) sign of the mean position errors indicated that in the horizon condition, subjects were less likely to respond to information indicating that they were drifting forward. The absolute value of the mean error indicated that the magnitude of these overall drift errors was significantly less in the $-18^{\circ}$ and the $-45^{\circ}$ conditions than in the horizon condition. A similar trend characterized the standard deviation of position error, which measured how effectively subjects detected and responded to the simulated gusts; better performance was observed in the $-45^{\circ}$ and $-18^{\circ}$ conditions than in the horizon condition.

Although Experiment 1 did not demonstrate unequivocally that greater gaze-movement angles support superior position control, it did show that subjects could extract sufficient information in the $-18^{\circ}$ and $-45^{\circ}$ conditions to perform the position control task at least as well as in a condition in which the camera pointed continuously toward the horizon (and in which pursuit fixation was not simulated). If we consider the nature of the visual information available in the two simulated-pursuit (i.e., the $-18^{\circ}$ and $-45^{\circ}$ ) conditions, there is more than one reasonable explanation to account for this.

The improvement might have resulted directly from the manipulation of simulated gaze direction, as predicted, in which case subjects were able to respond more sensitively to the geometric image transformations that were visible at the steeper camera angles in the simulatedpursuit conditions. The image transformations that are most discernible when the forward-moving camera or eye pursues an element on the ground ahead are expansion and deformation; ${ }^{8}$ as the camera progresses forward and pans downward, the image expands overall while elongating in the vertical dimension. For a forward-moving camera, expansion and deformation both increase as the gaze angle moves downward from the horizon until they peak at a pitch angle of $-45^{\circ}$ (Koenderink \& van Doorn, 1976). If subjects used these transformations in performing the control task, this would lead us to expect subjects' sensitivity to the gusts to improve as the camera's pitch angle is increased, reaching a peak in the $-45^{\circ}$ condition. This would imply better performance in the $-45^{\circ}$ than in the $-18^{\circ}$ condition, but Experiment 1 might have failed to register a significant difference because our performance measures were not sufficiently sensitive.

A second possible explanation for the superior performance in the $-18^{\circ}$ and $-45^{\circ}$ conditions is that simulated pursuit isolated some aspect of the geometric image transformations that was obscured or was more difficult to pick up in the absence of simulated pursuit. This explanation is consistent with the conclusions of Cutting et al. (1992), who determined that under certain conditions, simulated-pursuit displays support better gaze-heading discriminations than displays without simulated pursuit.
If simulated-pursuit fixation does isolate some visual transformation that is useful for position control, we would expect subjects to perceive gust displacements more accurately under simulated-pursuit conditions than under display conditions matched for gaze-movement angle that do not include simulated pursuit. Experiment 1 did not test this explicitly, however, because gaze-movement angle was not manipulated independently of simulated pursuit; no comparison was made between simulatedpursuit displays and displays matched for gaze-movement angle that did not include simulated pursuit.

The opposite condition (namely, that displays without simulated pursuit support performance superior to that obtained with simulated pursuit) is an additional possibility. This would parallel existing findings regarding heading perception under simulated-pursuit conditions (Banks et al. 1996; Beusmans, 1994; Royden et al., 1992; Royden et al., 1994) and is not ruled out by the data in Experiment 1 . As stated earlier, it is possible that the $-18^{\circ}$ and $-45^{\circ}$ conditions supported superior performance solely because of the information yielded by gaze-movement angles, which were steeper than those in the horizon condition. It might be that an additional performance gain could be obtained by making available extraretinal eye movement information that is consistent with the depicted viewpoint displacements. This information is not available under simulated-pursuit conditions. To the extent that observers are subject to errors such as the curved-path misperception when pursuit fixation is accomplished by the display, not the oculomotor system (Banks et al., 1996), subjects should perceive gust displacements more accurately without simulated pursuit.

As noted, however, this was not tested in Experiment 1 because gaze-movement angle and simulated pursuit were not manipulated independently; both conditions with pitched camera orientations included simulated pursuit, whereas the condition with the near-zero gaze-movement angle did not. Experiment 1 did not, therefore, test whether making appropriate extraretinal information available in the conditions with pitched camera orientations (and therefore greater simulated gaze-movement angles) would yield an additional gain in performance. In order to address this limitation, both camera pitch and the availability of extraretinal eye movement information were manipulated as independent variables in Experiments 2 and 3. Experiment 2 served as a pilot experiment for separating the two factors.

\section{EXPERIMENT 2 Varying Camera Orientation and Simulated Pursuit Independently}

In Experiment 2, the manipulation of the camera's pitch angle between $-45^{\circ}$ and $-18^{\circ}$ was repeated to test whether significant performance differences would emerge with practiced subjects. In addition, performance in simulatedpursuit conditions was compared with that in matched conditions that displayed the same parts of the scene 
without simulating pursuit (no-simulated-pursuit conditions). A $2 \times 2$ crossed design was used that combined camera pitch $\left(-45^{\circ}\right.$ vs. $\left.-18^{\circ}\right)$ and pursuit (simulated vs. no-simulated) manipulations.

As in Experiment 1, the predicted outcome for the camera pitch manipulation was that control performance in the $-45^{\circ}$ conditions would exceed performance in the $-18^{\circ}$ conditions; greater sensitivity to fore-aft displacement was expected in both the $-45^{\circ}$ simulated-pursuit and the $-45^{\circ}$ no-simulated-pursuit conditions because of their steeper gaze angles, and because the display would present geometric transformations of greater magnitude in this orientation. Furthermore, in no-simulated-pursuit conditions, optic flow speeds were higher with the $-45^{\circ}$ than with the $-18^{\circ}$ configuration because of the ground's greater proximity. This could enable greater sensitivity to fore-aft displacements in the $-45^{\circ}$ no-simulated-pursuit conditions in either of two possible cases. If subjects were successful in following the instruction to keep their eyes stationary in the center of the display, the $-45^{\circ}$ nosimulated-pursuit displays would yield greater retinal slip resulting from each gust. Alternatively, if subjects allowed themselves to move their eyes and pursue texture on the ground and if extraretinal information is important in controlling locomotion (see below), the $-45^{\circ}$ nosimulated-pursuit displays would enable greater sensitivity because each gust would require faster ocular rotation.

The outcome of the simulated-pursuit manipulation depended on the importance of extraretinal eye movement information for performing the hover control task. If optimal performance requires the availability of extraretinal information that is consistent with the depicted movements in the display, subjects should perform better in the no-simulated-pursuit than in the simulated-pursuit conditions, because the latter displays do not make this information available. Alternatively, if visual information is sufficient for position control in the absence of extraretinal information, the simulated-pursuit manipulation should have no effect on performance.

\footnotetext{
Method

Subjects. Because the session effect in Experiment 1 indicated that training benefited performance, all but 1 of the subjects were run again. The 12 th subject, a male, was replaced by a female graduate student who had participated previously as a pilot subject. The experiment was completed in one 2.5 -h session.

Stimuli. Displays resembled those in Experiment 1, with slightly modified gust histories. There was a 5-sec no-gust interval before each trial, during which the subject could examine the view from the position to be maintained. After this, the gusts increased to full strength over a further 5 -sec interval before the 100 -sec trial interval. Forward-backward gusts were weakened slightly to shorten the contours' between-frame displacements and to reduce flicker. Peak speed was $\pm 1.16 \mathrm{~m} / \mathrm{sec}$ for the two lowest frequency forwardbackward components and $\pm 0.52 \mathrm{~m} / \mathrm{sec}$ for the remaining components. Altitude gust power was as in Experiment 1.

There were two manipulations: camera pitch angle and simulated pursuit (Figure 3 ). In the $-18^{\circ}$ conditions, the camera began each trial pointing toward a ground location 3.0 eye-heights in front of the eye point. In the $-45^{\circ}$ conditions, the camera began each trial pointing toward a ground location 1.0 eye-height ahead. In the no-
}

simulated-pursuit displays, the pitch angle remained constant at $-18^{\circ}$ (arctan 1 eye-height $/ 3$ eye-heights) or $-45^{\circ}(\arctan 1 / 1)$ throughout the trial. In simulated-pursuit displays, the camera panned up and down as it moved, which maintained at the screen's center the image of the ground location that was 3.0 or 1.0 eyeheights ahead of the camera position at the start of the trial.

Design. The same measures were used as in Experiment 1. Presentation order of the four conditions was partially counterbalanced across subjects. Each of four orders began with a different condition and was presented to 3 subjects. The sequence of simulatedpursuit and no-simulated-pursuit conditions was 1221 or 2112 in all orders, whereas the sequence of $-45^{\circ}$ versus $-18^{\circ}$ conditions was 1122 or 2211 . In an attempt to reduce variability, the number of repetitions in each condition was increased to seven. Means were calculated across repetitions for each dependent measure and were analyzed in a $2\left(-45^{\circ}\right.$ vs. $\left.-18^{\circ}\right) \times 2$ (simulated-pursuit vs. nosimulated-pursuit) within-subjects design.

Apparatus. Hardware and software were as in Experiment 1.

Procedure. Instructions reviewed the position control task. Before $-18^{\circ}$ no-simulated-pursuit trials, subjects were instructed that the camera would point slightly below the horizon and remain at this slightly pitched orientation throughout the trial. Before $-45^{\circ}$ no-simulated-pursuit trials, instructions stated that the camera would point forward and down at a constant steep angle that would reveal the ground nearby. Before $-18^{\circ}$ simulated-pursuit trials, subjects were informed that the camera would point toward a location on the ground some distance ahead and would pivot up and down to keep this point centered on the screen. Before $-45^{\circ}$ simulatedpursuit trials, subjects were instructed about the camera's simulated pursuit of a nearby fixation point. Subjects completed five practice trials before each condition.

\section{Results and Discussion}

Cell means are shown in Figure 4. No significant effects on mean position error of camera pitch $[F(1,11)=$ $2.45]$, simulated pursuit $[F(1,11)=4.57]$, or interaction between the two factors $[F(1,11)=1.47]$ were observed. Similarly, no significant camera pitch $[F(1,11)=.19]$, simulated pursuit $[F(1,11)=2.12]$, or interaction $[F(1,11)=$ $.15]$ effects were observed for the absolute values of the mean error. Camera pitch did influence the standard deviation of the position error $[F(1,11)=5.68, p<.05]$, with superior performance observed in the $-45^{\circ}$ conditions. There was no effect of simulated fixation on the standard deviation of position error $[F(1,11)=.81]$; neither was there a significant interaction $[F(1,11)=.14]$.

Subjects maintained position more effectively when the camera pointed toward a ground location 1 eye-height ahead than when it faced the more distant location. This modest improvement manifested itself solely in the standard deviation of the position error; as in Experiment 1, there was no significant difference in mean errors, or in error absolute values, between the $-45^{\circ}$ and the $-18^{\circ}$ conditions.

This improvement appears to indicate that the camera's pitch angle was a more important determinant of control performance than the inclusion of simulated pursuit, and that dynamic visual information is sufficient to support optimal position control even when this information is not accompanied by eye movements appropriate to the depicted movements. Drawing this conclusion would be premature, however. There were several limitations in 


\section{-18 deg conditions: The camera is oriented to reveal a distant location}

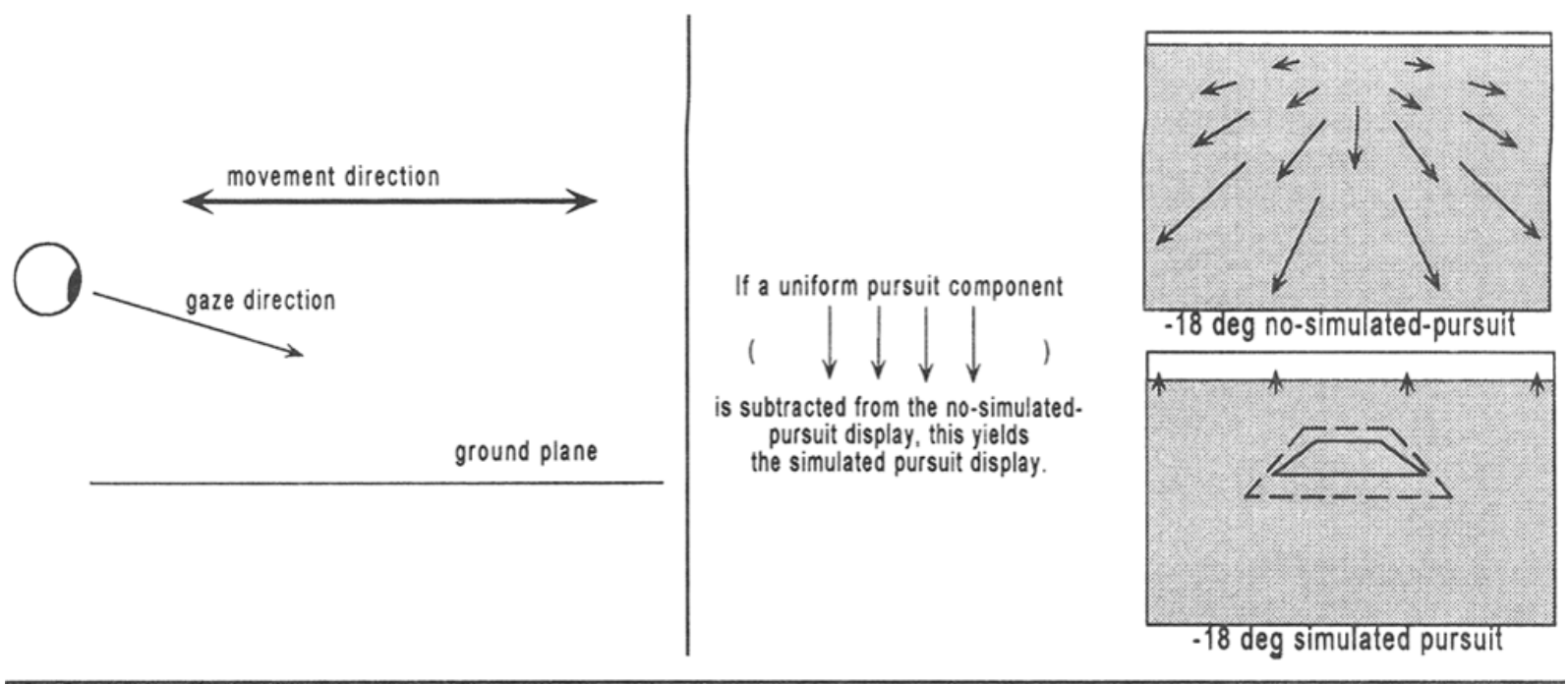

-45 deg conditions: The camera is oriented to reveal a nearby location

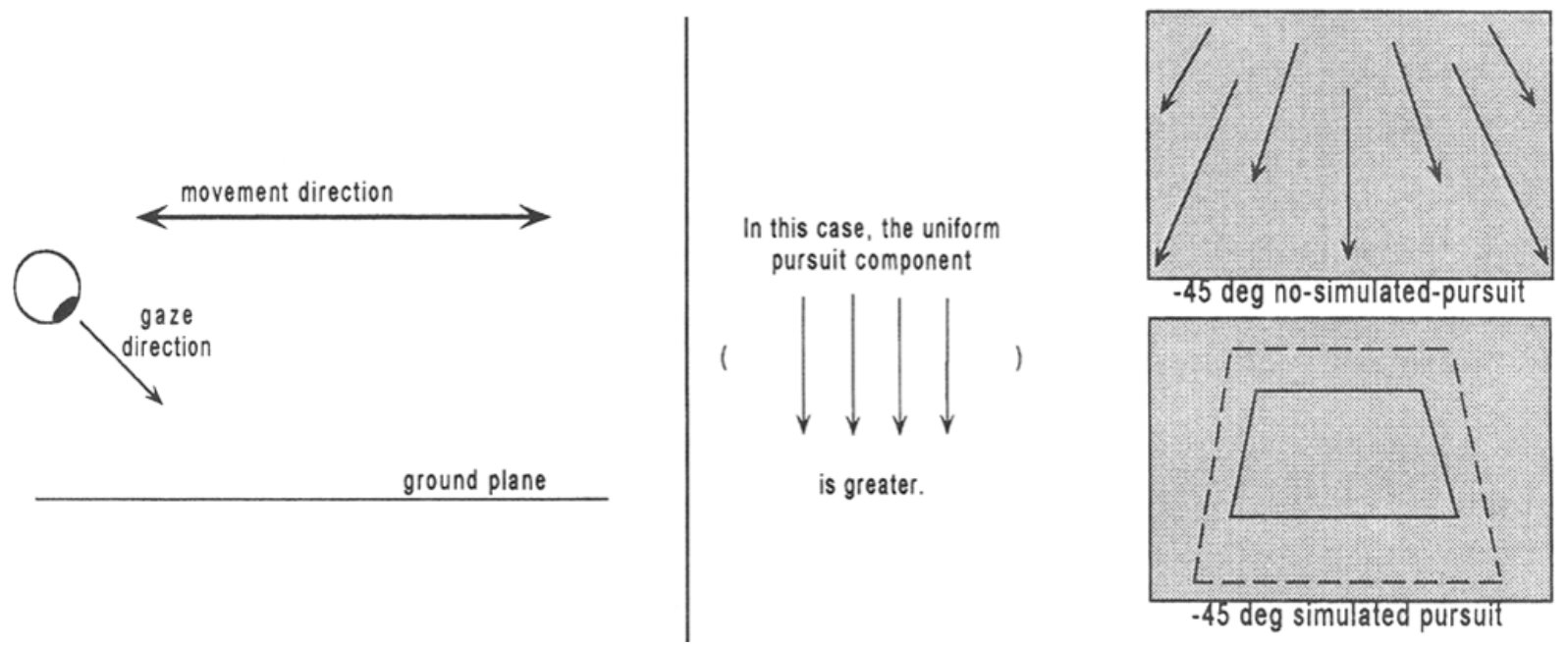

Figure 3. Layout of the camera pitch and pursuit conditions in Experiment 2. Upper panel: $-18^{\circ}$ no-simulated-pursuit and $-18^{\circ}$ simulated-pursuit conditions. Lower panel: $-45^{\circ}$ no-simulated-pursuit and $-45^{\circ}$ simulated-pursuit conditions. (Figure is not to scale. Left panels show side views. Right panels show screen displays; subtracting the pursuit component from the no-simulated-pursuit flow field yields the simulated-pursuit flow field.)

the design of Experiment 2 that might have obscured the possible benefits of making appropriate extraretinal eye movement information available.

The first limitation was the relative imprecision with which the subjects' gaze was controlled in Experiment 2. Although subjects had been instructed to keep their gaze near the center of the display, no fixation target was displayed. This problem was aggravated by a lack of precision in the instructions, which failed to tell subjects to fixate a specific feature on the ground or, alternatively, to keep their eyes immobile while "maintaining gaze near the center of the screen." As a consequence, eye movements were unconstrained in simulated-pursuit conditions; subjects might have been moving their eyes in the condition that was designed to eliminate the availability of extraretinal eye movement information. (This does not change the fact that the simulated-pursuit conditions did not make available appropriate extraretinal eye movement information; even if subjects were making eye movements, these movements could not have been consistent with the depicted camera displacements.) Conversely, the lack of a fixation target and of specific instructions compromised the effectiveness of the no-simulated-pursuit conditions, whose moving texture makes them appropriate for testing the possible beneficial effects of extraretinal information.

The second limitation in Experiment 2 was that the uniform checkerboard ground texture created an artifact that might have hurt performance in the no-simulatedpursuit conditions. If the subject became distracted while 
Exp. 2: Mean position error

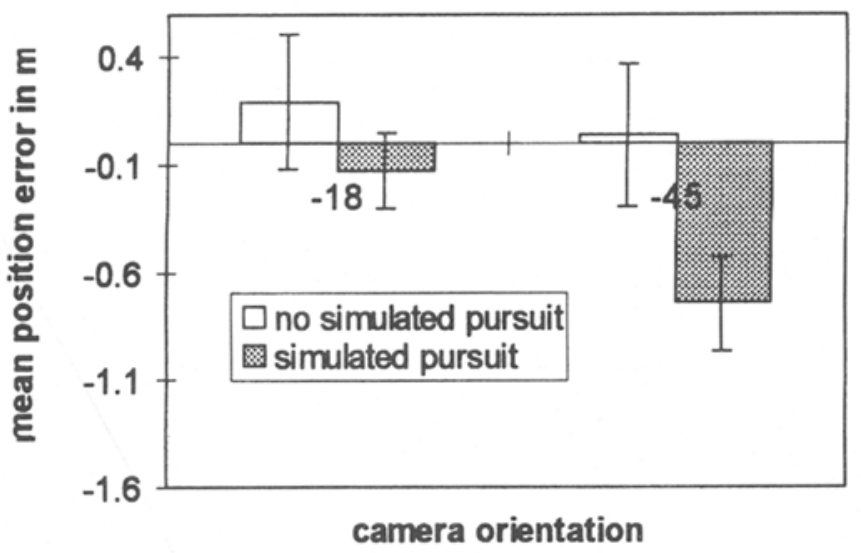

Exp. 2: Absolute value of mean position error

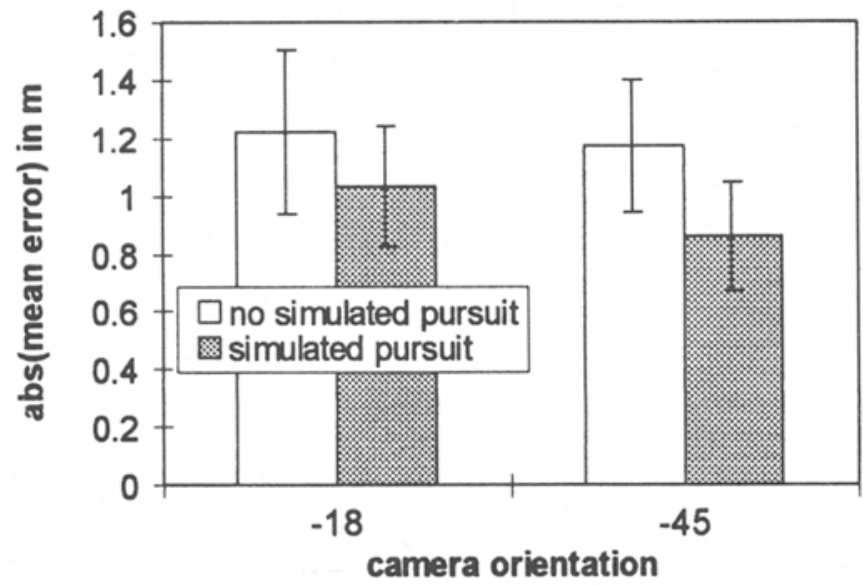

Exp. 2: Standard deviation of position

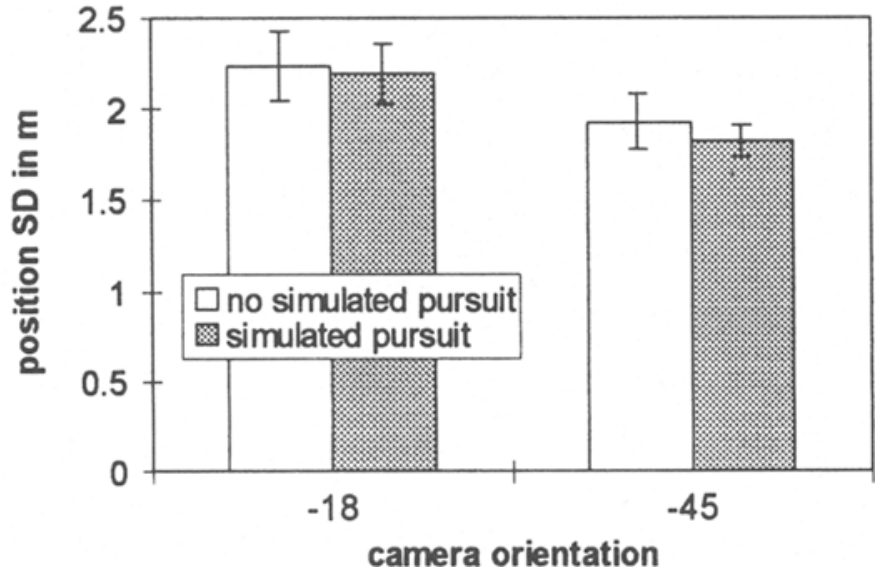

Figure 4. Upper panel: mean position error in Experiment 2. Middle panel: absolute values of mean position error, Experiment 2. Lower panel: standard deviations of position error, Experiment 2. (Values are means across subjects and repetitions, displayed by condition. Units are meters. Error bars represent 1 SEM.) 
fixating a given patch of texture at the center of the screen, a strong gust might have moved the camera forward or backward, thus introducing new texture to the center of the screen. If the subject was unaware of this (because all the squares looked the same), he/she would have continued the task, working to maintain a new position that might have been several squares away from the original. The resulting increase in position errors would have been restricted to the no-simulated-pursuit conditions; in simulated-pursuit conditions, the display always followed the same piece of texture, which made such drift errors unlikely.

The third limitation in Experiment 2 was its inclusion of only two camera orientations. Although it was shown that pitching the camera downward $45^{\circ}$ as opposed to $18^{\circ}$ reduced variable control error, this did not eliminate the possibility that other camera orientations might have supported even better performance. This argued for a more thorough examination of visual position control performance, evaluated over a wider range of viewing angles. Experiment 3 was conducted to address these limitations and to test more rigorously the camera orientation and oculomotor-pursuit conditions that would support optimal performance in the position control task.

\section{EXPERIMENT 3 \\ Camera Orientation and Eye Movement Information Interact to Determine Position Control Performance}

Experiment 3 tested systematically the effects on position control of varying gaze-movement angle and the availability of extraretinal eye movement information. A new display was constructed in order to address the first two limitations that were identified in Experiment 2. This included a modified texture pattern that allowed better control over the subject's gaze and reduced the possibility of drift artifacts. Instead of a checkerboard of homogeneous squares, the ground texture was an array of triangles lying flat on the ground in random orientations. In the midst of this array, a single triangle was highlighted. Subjects were instructed to fixate this target, which was located at the center of the screen at the beginning of all trials. Throughout trials in the simulated-pursuit conditions, the fixation target remained stationary at the center of the screen. In the conditions in which pursuit was not simulated, the fixation target and therefore subjects' gaze $^{9}$ moved on the screen in response to the camera's excursions; for this reason, these conditions were called "real-pursuit conditions." The movements of the target in real-pursuit displays were optically slow when the camera pointed toward distant terrain and rapid when the camera revealed nearby terrain.

The addition of a fixation target and the improved instructions made it possible to evaluate the effects of varying gaze-movement angle and the availability of consistent extraretinal eye movement information more effectively than in Experiment 2. (Because of these im- provements, the manipulation of camera orientation is referred to explicitly as the "gaze-movement angle manipulation" in Experiment 3.) Since subjects were instructed to fixate the target whether or not it was moving, the ambiguity that was present in the Experiment 2 nosimulated-pursuit conditions between immobile fixation (which would yield retinal slip combined with extraretinal signals indicating no eye movement) and pursuit of a location on the ground (which would yield eye movement information consistent with the depicted gusts) was eliminated. In addition to increasing experimental control over the subject's gaze, instructing subjects to fixate a distinct target in a nonuniform texture field eliminated the possibility that they might lose track of where they were supposed to be looking (and maintaining position) in real-pursuit conditions.

In order to address the third limitation that was identified in Experiment 2, a wider range of camera orientations was included in Experiment 3 . The boundary pitch values were $-15^{\circ}$, approximately equivalent to the $-18^{\circ}$ conditions in Experiments 1 and 2, and $-90^{\circ}$, in which the camera pointed down at the ground directly below it. Two intermediate conditions of $-40^{\circ}$ and $-65^{\circ}$ pitch bridged these boundary orientations at equal-angle intervals.

As in Experiment 2, the relative importance of gazemovement angle and of extraretinal eye movement information should determine which condition supports the best performance. If subjects perform the position control task using only the geometric transformations available in the display (i.e., if purely visual information is sufficient to support optimal performance in the absence of appropriate extraretinal information), there should be no difference in performance between simulated-pursuit and real-pursuit conditions. In addition, superior performance should be obtained in both simulated-pursuit and real-pursuit conditions when the camera displays locations in the scene where geometric image transformations are most easily discernible. In these experiments, this implies aiming the camera toward a ground location 1 eye-height ahead, at which both expansion and deformation are maximal for a camera moving in the fore-aft dimension. In Experiment 3, this predicts superior performance in the $-40^{\circ}$ condition (which approximates most closely this ideal pitch orientation) in both simulatedpursuit and real-pursuit conditions.

If, on the other hand, subjects use extraretinal pursuit information in conjunction with flow information, performance should be impaired in simulated-pursuit relative to real-pursuit conditions. In real-pursuit conditions, moreover, performance should be related to the flow speed of the fixated object; this predicts superior performance in the $-90^{\circ}$ real-pursuit condition; optic flow is faster in this location than anywhere else in the optic array. It should be noted that this prediction rests on two assumptions: (1) The target's optic flow will not be too rapid for subjects to be able to follow the fixation instructions, and (2) faster oculomotor pursuit supports greater sensitivity to the gust movements. 


\section{Method}

Subjects. Because of limited subject availability, Experiment 3 was planned to include only 8 subjects but more practice than in Experiments 1 and 2. A 9th subject was added to one of the two counterbalanced groups; the benefit of this subject's unexpected availability was judged to be greater than the penalty of a slightly unbalanced order design. Two of the 9 subjects had served in Experiments 1 and 2 .

The experiment was conducted over four 1.5-h sessions. Each subject completed a full practice session in each set of pursuit conditions. Subjects completed a day of practice for one set of pursuit conditions (simulated pursuit or real pursuit, depending on the subject's group), and then a second, test day for those conditions. On the 3rd and 4th days, practice and test sessions, respectively, for the other set of pursuit conditions were conducted.

Stimuli. Display format was similar to that of Experiment 2, with several modifications. The display was switched to a rear-projection layout incorporating a screen $1.8 \mathrm{~m}$ high and $2.4 \mathrm{~m}$ wide. Viewing position was at the projection center, $1.2 \mathrm{~m}$ from the screen, yielding an image $74^{\circ}$ high and $90^{\circ}$ wide.

The checkerboard ground texture used in the earlier experiments was replaced by a similarly sized array of dark-green equilateral triangles lying flat on a light-green ground (Figure 5). The number of texture elements was the same as in the earlier checkerboard. One triangle was white and was designated as the fixation target. Triangles were $0.91 \mathrm{~m}$ on a side and were oriented randomly. They were sufficiently large to be distinguishable as triangles even when viewed from some distance in the scene. As a result, the fixation target projected a fairly large image in the $-90^{\circ}$ conditions; if a gust were to push the camera downward from its initial altitude to a 3-m altitude, for example, the edge of the image of the target triangle would be $4.9^{\circ}$ from the center. The fixation target was situated at the center of the screen at the beginning of all trials. The target remained stationary throughout simulated-pursuit displays, whereas it moved up and down on the screen in response to camera movements in the real-pursuit conditions. The time course of the trials and the gust histories was the same as in Experiment 2. Four camera orientations were displayed: $-15^{\circ},-40^{\circ},-65^{\circ}$, and $-90^{\circ}$. At the beginning of each $-15^{\circ},-40^{\circ},-65^{\circ}$, and $-90^{\circ}$ trial, the fixation target was 3.7 , $1.2,0.47$, and 0.0 eye-heights ahead, respectively. Gaze attitude varied considerably throughout trials in which the camera pointed toward nearby terrain (i.e., $-40^{\circ},-65^{\circ}$, and $-90^{\circ}$ trials). For example, only a small forward gust would be required in $-40^{\circ}$ conditions to bring about a momentary gaze attitude of $-45^{\circ}$ before the subject's position correction. For this reason, both the $-40^{\circ}$ and the $-65^{\circ}$ conditions can be regarded as approximating the $-45^{\circ}$ gaze configuration at which divergence and deformation are maximal for forward-backward movement. The simulated-pursuit manipulation was similar to that of Experiment 2.

Design. Presentation order was partially counterbalanced across and within two groups of subjects. The first group completed practice and test sessions for the real-pursuit conditions on Days 1-2 and practice and test sessions for the simulated-pursuit conditions on Days 3-4, whereas the second group completed the conditions in the reverse order. In each group, the camera orientation conditions were presented in one order in the practice sessions and the reverse order in the test sessions. Practice and test sessions included seven repetitions for each condition. Means were calculated across repetitions for each dependent measure and were analyzed in a 4 (gaze-movement angles) $\times 2$ (simulated vs. real-pursuit) withinsubjects design.

Apparatus. Hardware and software were as in Experiment 2, except the Electrohome projector was switched to a rear-projection configuration. The image was projected onto an Optixx Polylens screen incorporating an $\mathrm{F} / 1.2$ Fresnel lens with 55 ridges/radial inch.

Procedure. Apart from the addition of the fixation target, instructions resembled those in the earlier experiments.

\section{Results and Discussion}

Cell means for the position error means, their absolute values, and the error standard deviations are shown in Figure 6. No significant effects on mean position error of gaze-movement angle $[F(3,24)=1.68]$, simulated pursuit $[F(1,8)=1.12]$, or interaction between the two factors $[F(3,24)=1.49]$ were observed.

There was a main effect of gaze-movement angle on the absolute values of the mean error $[F(3,24)=7.64, p<$ $.001]$. Pairwise Bonferroni contrasts identified as significant the difference between the $-15^{\circ}$ camera orientation and the $-65^{\circ}$ and $-90^{\circ}$ orientations. Although the simulated-pursuit factor did not affect the error absolute value $[F(1,8)=1.31]$, there was an interaction between camera angle and pursuit $[F(3,24)=5.19, p<.01] . T$ tests between cell means (two-tailed, $\alpha=.05$ ) indicated that the error absolute values were greater in the simulatedpursuit than in real-pursuit displays at the $-65^{\circ}$ and $-90^{\circ}$ orientations. Error absolute values for real-pursuit displays at the $-15^{\circ}$ orientation were significantly greater than at the remaining three orientations.

Camera orientation influenced the standard deviation of position error $[F(3,24)=26.4, p<.0001]$. Bonferroni contrasts identified significant differences between the $-15^{\circ}$ orientation and the remaining three orientations. The inclusion of simulated pursuit influenced the position error standard deviation $[F(1,8)=5.41, p<.05]$. There was an interaction between gaze angle and pursuit $[F(3,24)=$ $22.2, p<.0001]$. Between-cell $t$ tests indicated that the error standard deviation values were greater in simulatedpursuit than in real-pursuit displays at the $-65^{\circ}$ and $-90^{\circ}$ orientations. In contrast, standard deviation error values were significantly greater in the real-pursuit than in the simulated-pursuit displays at the $-15^{\circ}$ orientation. ${ }^{10}$ Error standard deviation values for real-pursuit displays at the $-15^{\circ}$ orientation were significantly greater than at the remaining three orientations, and standard deviation values in the $-40^{\circ}$ real-pursuit condition were greater than in the $-90^{\circ}$ real-pursuit condition.

Experiment 3 was a more rigorous test than either Experiment 1 or 2 of the relative importance of gaze-movement angle and of extraretinal eye movement information for position control. Results indicated that both factors influenced control performance. A distinguishing feature of the data can be noted selectively in the real-pursuit conditions, in which the means for all three error measures showed progressive decreases as the camera's downward pitch angle was increased. Although this trend was not identified as statistically significant for the signed mean of the position error, it was significant for the two other measures. The (unsigned) absolute value of the mean error indicated that subjects had a greater tendency to drift when gaze angles were shallower and more distant terrain was shown. The decrease in the magnitude and variability of this measure at steeper pitch angles in the real-pursuit conditions could be explained as a result of subjects' using extraretinal eye movement information when the camera was pointing at nearby terrain. The error absolute value 

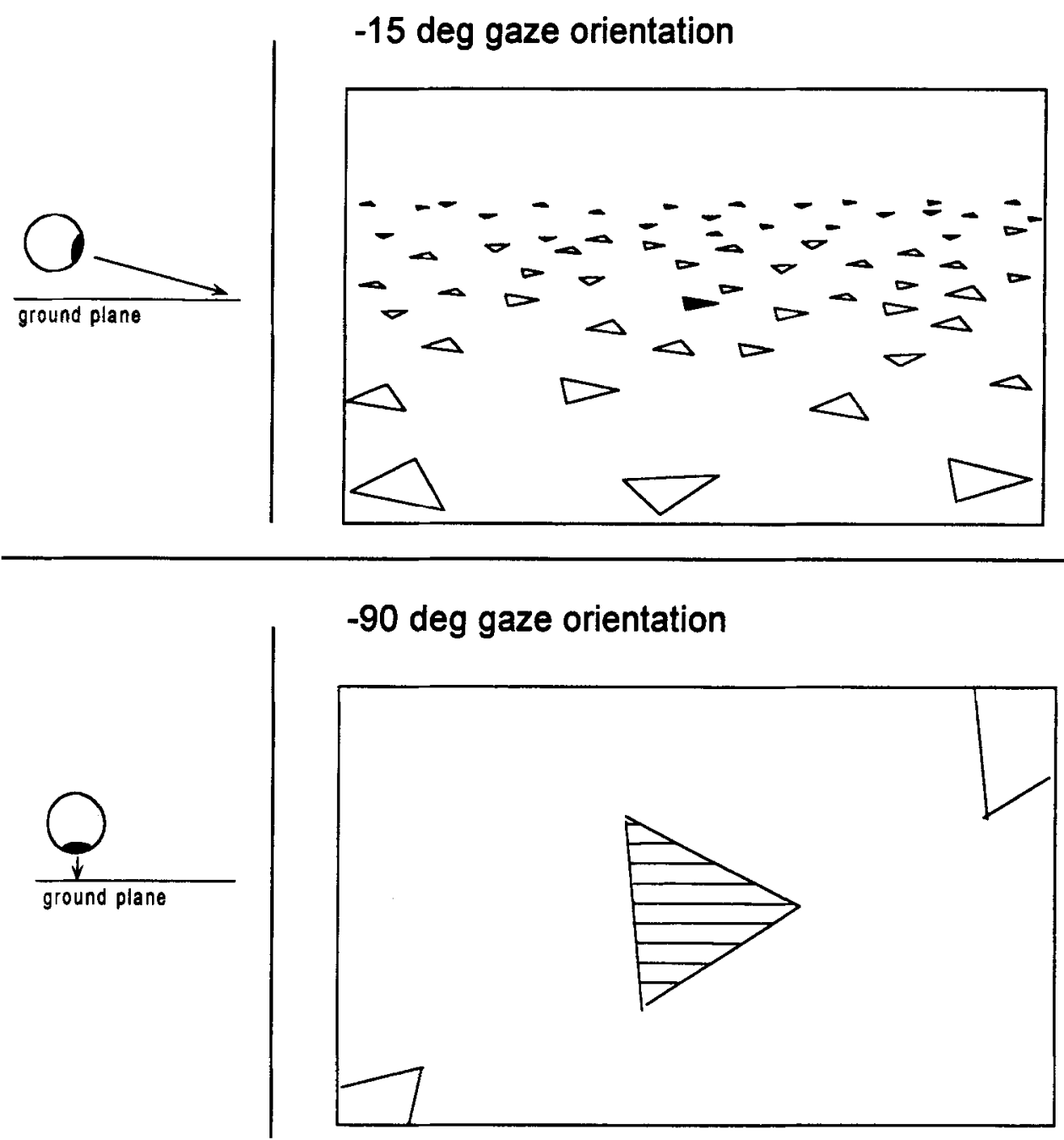

Figure 5. Layout for the shallowest $\left(-15^{\circ}\right)$ and steepest $\left(-90^{\circ}\right)$ gaze orientation configurations in $\mathrm{Ex}-$ periment 3. (Figure is not to scale. Left panels show side views. Right panels represent the screen displays. The marked triangle is the fixation target.)

measure indicates in addition that the slightly lesser drift values observed in the simulated-pursuit conditions in Experiment 2 were indeed an artifact, caused by subjects' occasionally losing track of their target position in conditions in which pursuit was not simulated.

The observed effect of gaze-movement angle on the standard deviation of position indicated that subjects performed better and less variably at steeper orientations, which pointed the camera toward nearby, fast-flowing terrain. Assuming that subjects were successful in following the fixation instructions, this improvement is notable because it was due largely to the availability of appropriate extraretinal information, which varied not only with the inclusion of simulated pursuit, but also with the proximity (and therefore the optical speed) of the fixated element in the scene. Evidence for this conclusion is found in the continued drop in standard deviation error values with increased pitch angle in the real-pursuit conditions; the availability of appropriate extraretinal eye movement information apparently enabled better performance in the real-pursuit conditions than in the simulatedpursuit conditions at the steepest camera angles. Importantly, performance was better in the $-90^{\circ}$ real-pursuit condition than in the $-40^{\circ}$ real-pursuit condition, in which were displayed geometric image transformations of the highest magnitude of the four orientation conditions.

The simulated-pursuit conditions, in contrast, yielded standard deviation error performance that was poorer overall than that in the real-pursuit conditions and failed to replicate the small improvement in the standard deviation error measure that was observed to accompany the increase in gaze-movement angle in Experiment 2. In addition, none of the measures identified a peak in performance in the simulated-pursuit conditions at the $-40^{\circ}$ gaze orientation, as would be expected if subjects were relying only on visual image transformations independent of extraretinal eye movement information. These findings are consistent with the results of Experiment 1, in 
Exp. 3: Mean position error

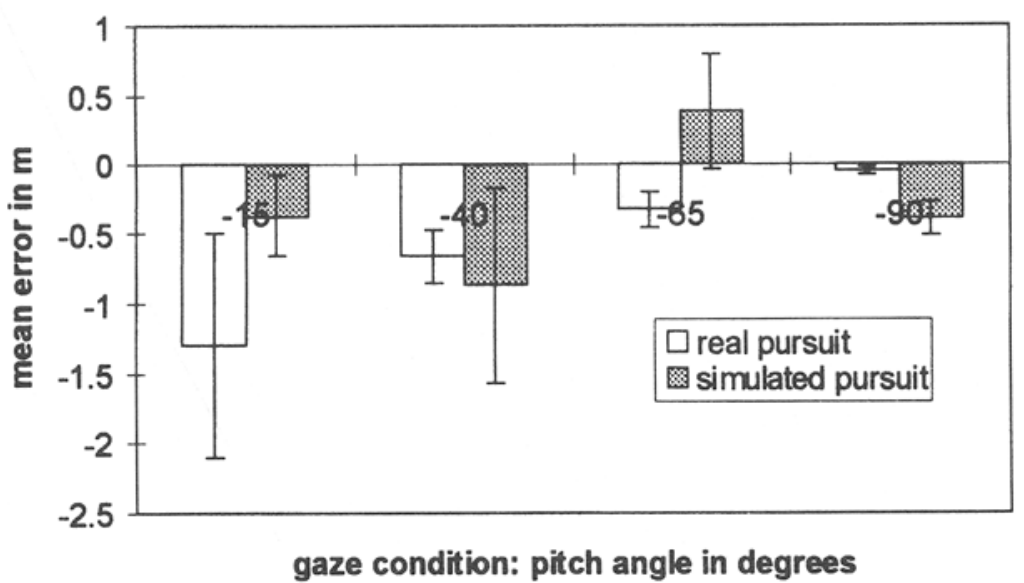

Exp. 3: Absolute value of mean position error

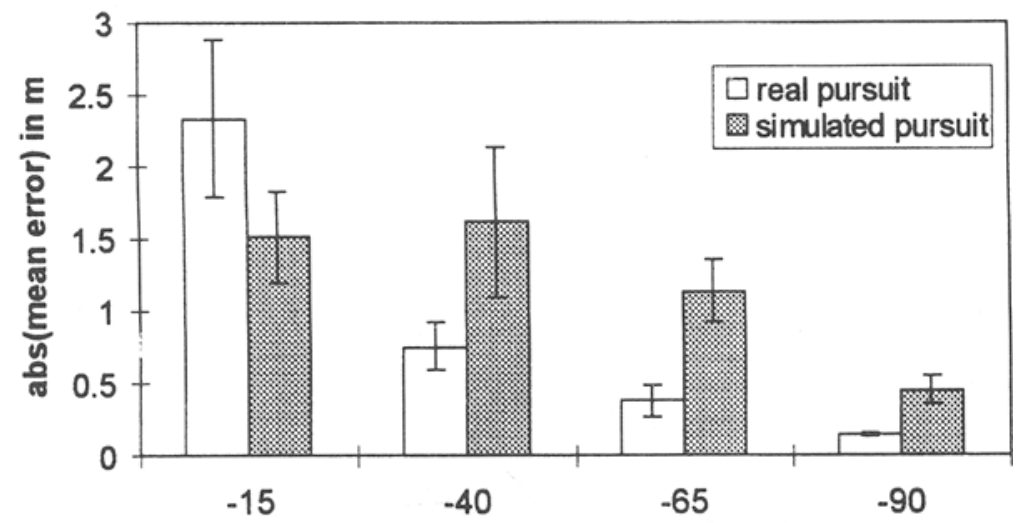

gaze condition: pitch angle in degrees

Exp. 3: Standard deviation of position

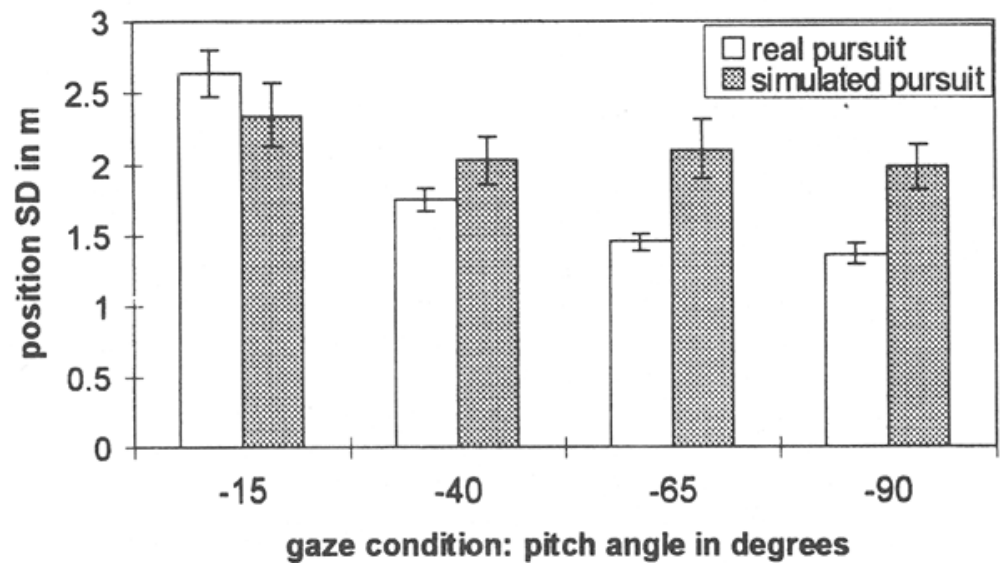

Figure 6. Upper panel: mean position error in Experiment 3. Middle panel: absolute values of mean position error, Experiment 3. Lower panel: standard deviations of position error, Experiment 3. (Values are means across subjects and repetitions, displayed by condition. Units are meters. Error bars represent $1 S E M$.) 
which no significant performance difference was observed between conditions displaying distant versus nearby simulated fixation locations.

It is worthwhile to consider several aspects of the displays that might have influenced the observed effects. First, in the $-90^{\circ}$ displays, it is unlikely that subjects were fixating the exact center of the target triangle throughout each trial. This is because the target image was large and lacked internal features, and subjects' gaze might have drifted around within the triangle or followed one of the vertices. This does not, however, invalidate the comparison between the real-pursuit conditions (where the visual information was consistent with whatever extraretinal tracking information the subject might have used) and the simulated-pursuit conditions (where the two information classes were inconsistent).

The second issue is that in $-90^{\circ}$ real-pursuit displays, subjects could have performed the task simply by keeping the target centered on the screen (thus keeping the camera above the target independent of altitude). It is not unreasonable that this cue might have helped subjects minimize drift, and these trials did yield the lowest, least variable position error means and absolute values. The gaze angle effect extended also, however, to the variable standard deviation error measure, which tested the subject's ability to counter gusts as they occurred, and it seems unlikely that this could have resulted solely from a visual symmetry cue. If symmetry were the only basis for the improvement in standard deviation error, we would expect a performance improvement only at $-90^{\circ}$; we would not expect better performance in the $-65^{\circ}$ and the $-40^{\circ}$ real-pursuit trials than in the $-15^{\circ}$ real-pursuit trials (which was observed), and we would expect a significant difference between the $-65^{\circ}$ and the $-90^{\circ}$ realpursuit conditions (which was not observed).

Third, it is notable that in contrast to the poorer performance found in simulated-pursuit conditions when the camera was oriented to show nearby terrain, there was a small improvement in the standard deviation of position error in simulated-pursuit relative to the real-pursuit displays at the $-15^{\circ}$ orientation (with which the camera pointed toward the most distant terrain). This performance benefit might have resulted directly from the continuous visibility of the horizon in the $-15^{\circ}$ displays; since the gusts caused the horizon to move in the simulatedpursuit displays, this might have provided an additional dynamic position cue. This observed benefit was clearly less than the reverse performance disparity that was observed at the $-65^{\circ}$ and $-90^{\circ}$ orientations. Moreover, in Experiment 2, no analogous performance difference was identified between the simulated-pursuit and the nosimulated-pursuit displays in the $-18^{\circ}$ camera configuration. For these reasons, we cannot from these data conclude unequivocally that simulated pursuit benefits position control when the camera is pointing at distant terrain.

Lastly, it is also notable that in Experiment 3 (as had occurred in Experiments 1 and 2), several subjects commented during simulated-pursuit displays that when the camera moved forward and panned downward, they felt as if they were nosing into the ground on a curving path. This suggests that subjects were confounding the visual consequences of forward and downward-veering movement, much as subjects have confounded the consequences of forward and laterally veering movement in heading studies that included simulated pursuit (Banks et al., 1996; Royden, 1994; Royden et al., 1992; Royden et al., 1994). One way to characterize subjects' performance in Experiment 3 and the occurrence of curved-path misperceptions in simulated-pursuit conditions is in terms of signal and noise components that are, respectively, useful or irrelevant to the control task (Flach et al., 1992; Garness et al., 1994). In these experiments, image transformations resulting from forward or backward gusts constituted a signal component, whereas transformations associated with any other movements constituted noise activity. As noted, fore-aft movement causes signal image transformations, including deformation, which conveys changes in the ground's slant with respect to the gaze axis, and expansion or contraction, which convey approach or recession, respectively, of the surface. The superior performance observed in real-pursuit conditions in Experiment 3 is consistent with subjects' ability to perceive linear selfmotion accurately during ocular-pursuit rotations in the real world and indicates that these signal transformations are best utilized for performing the control task when they are accompanied by appropriate eye movements. In simulated-pursuit displays, by comparison, the signal transformations still indicate slant and distance changes, while extraretinal information is indicating that the eyes are stationary in the head. An explanation for the increased control error in simulated-pursuit conditions and for the reports of curved-path misperception is that subjects responded to the combination of signal transformations and extraretinal information indicating no eye movement as a sign of a curvilinear path confounded by an illusory, veering noise component. ${ }^{11}$

\section{GENERAL DISCUSSION}

Three experiments tested the effects of gaze direction and the availability of extraretinal eye movement information on performance in a visual position control task. The pitch orientation of the virtual camera and the inclusion of simulated pursuit fixation, respectively, were used to probe the two factors' effects on position control performance.

Before discussion of the findings in Experiments 1-3, two qualifications should be noted. First, these findings are more closely relevant to the demands of low-speed than of high-speed locomotion, where optic flow speeds and the magnitudes of the geometric image transformations are much greater. The task demands of planning and modulating self-motion differ between subjects moving rapidly through a scene at many eye-heights per second, and subjects hovering in or walking through a scene. In particular, Experiments 1-3 do not countermand the "look where you want to go" rule on the road (Bondurant 
\& Blakemore, 1982); if truck drivers began trying routinely to fixate a point 1 eye-height ahead or nearer on the highway, the results would be unfortunate.

Second, the availability or lack of vestibular and other dynamic sensory information sources may complicate the issue of how subjects control self-motion while fixating a point on the ground. Whereas Experiments 1-3 tested performance on a self-motion control task in an environment offering only visual input, the nonvisual senses offer additional and in some cases more immediate registration of translational and rotational accelerations. In the vestibulo-ocular reflex, for example, dynamic input enables the maintenance of retinal fixation when the viewer is subjected to movement at frequencies above those at which ocular corrections are made based on purely visual information (Gillingham \& Previc, 1993); this could influence the viewer's ability to extract and interpret visual transformations during self-motion through a scene comprising nearby objects and surfaces. For this reason, further analyses of locomotion control in this context should consider dynamic nonvisual mechanisms in addition to visual transformations.

Experiments 1-3 indicated that gaze-movement angle can influence control performance; when the angle separating the gaze direction and the primary, fore-aft axis of movement was increased by pitching the camera down from the horizon, indices of position control performance generally improved. In Experiment 1, subjects performed better in two simulated-pursuit conditions (in which the camera pursued either a distant or a nearby location on the ground) than in a condition without simulated pursuit in which the camera pointed toward the horizon. Experiment 2 repeated Experiment 1 's comparison between distant and nearby fixation locations, but included conditions both without and with simulated pursuit. Subjects performed slightly better (as indicated by a small decrease in the standard deviation of the position error) when the camera pointed at the nearby location in both classes of pursuit display. Although this suggested that subjects could perform the task using geometric transformations in the image regardless of whether the transformations were accompanied by appropriate eye movements, limitations were identified in the experimental design that indicated a need for a more rigorous test across a broader range of gaze orientations. This was accomplished in Experiment 3 , in which gaze-movement angle was again found to influence control performance as a main effect (as indicated by the absolute value of the mean position error and by the standard deviation of the position error).

Experiment 3 indicated, however, that this improvement in performance at steeper gaze orientations could not be attributed solely to increased gaze-movement angles or to the greater proximity of the terrain displayed on screen. The finding that the availability of extraretinal eye movement information interacted with gaze direction to influence control performance clarified the findings from Experiment 2, which were obtained with less precise control of the available visual and extraretinal information and with a narrower range of camera orientations.
Experiment 3 indicated that although subjects could use visual image transformations alone to control position adequately with a distant point of regard, they also used extraretinal eye movement information for optimal performance when they were viewing nearby terrain. The main reason for drawing this conclusion is that the performance improvement at greater gaze-movement angles was obtained largely in the real-pursuit conditions; in these displays, pursuit of the fixation target, a marking on the ground, was accomplished not by the display simulation but by the viewer's oculomotor system. Findings from Experiment 3 were interpreted as an indication that extraretinal information is important not only for extracting heading (Banks et al. 1996; Beusmans, 1994; Royden et al., 1992; Royden et al., 1994), but also for controlling one's position visually in the fore-aft dimension when one is hovering over a textured ground surface.

\section{REFERENCES}

Banks, M. S., Ehrlich, S. M., Backus, B. T., \& Crowell, J. A (1996). Estimating heading during real and simulated eye movements. Vision Research, 36, 431-443.

Beusmans, J. (1994). Perception of egomotion. In A. Liu \& J. Beusmans (Eds.), Cambridge Basic Research 1994 annual report (pp. 69). Cambridge, MA: Nissan Cambridge Basic Research.

Bondurant, R., \& Blakemore, J. (1982). Bob Bondurant on highperformance driving. Osceola, WI; Motorbooks International.

Crowell, J. A., \& Banks, M. S. (1993). Perceiving heading with different retinal regions and types of optic flow. Perception \& Psychophysics, 53, 325-337.

Cutting, J. E. (1986). Perception with an eye for motion. Cambridge, MA: MIT Press.

Cutting, J. E., Springer, K., Braren, P. A., \& Johnson, S. H. (1992). Wayfinding on foot from information in retinal, not optical, flow. Journal of Experimental Psychology: General, 121, 41-72.

Cutting, J. E., Vishton, P. M., FlüCKiger, M., Baumberger, B., \& GERNDT, J. D. (1997). Heading and path information from retinal flow in naturalistic environments. Perception \& Psychophysics, 59, 426-441.

Ehrlich, S. M., Beck, D. M., Crowei.l, J. A., Freeman, T. C. A., \& BANKS, M. S. (1998). Depth information and perceived self-motion during simulated gaze rotations. Vision Research, 38, 3129-3145.

Flach, J. M., Hagen, B. A., \& Larish, J. F. (1992). Active regulation of altitude as a function of optical texture. Perception \& Psychophysics, 51, 557-568.

Garness, S. A., Flach, J. M., Stanard, T., \& Warren, R. (1994). The basis for the perception and control of altitude: Splay and depression angle components of optic flow. Proceedings of the Human Factors and Ergonomics Society 38th Annual Meeting, 2, 1275-1279.

GiBson, J. J. (1950). The perception of the visual world. Boston: Houghton Mifflin.

GiBson, J. J. (1979). The ecological approach to visual perception. Boston: Houghton Mifflin.

Gillingham, K. K., \& Previc, F. H. (1993). Spatial orientation in flight (AL-TR-1993-002). Brooks AFB, TX: U.S.A.F. Armstrong Laboratory.

HILDRETH, E. C. (1992). Recovering heading for visually-guided navigation. Vision Research, 32, 1177-1192.

HoCHBERG, J. (1986). Representation of motion and space in video and cinematic displays. In K. R. Boff, J. P. Thomas, \& L. Kaufman (Eds.), Handbook of perception and human performance (Vol. 1, pp. 22.122.68). New York: Wiley.

Johnson, W. W., Tsang, P. S., Bennett, C. T., \& Phatak, A. V. (1989). The visually guided control of simulated altitude. Aviation, Space, \& Environmental Medicine, 60, 152-156.

Koenderink, J. J. (1986). Optic flow. Vision Research, 26, 161-180.

Koenderink, J. J., \& van DoORN, A. J. (1976). Local structure of movement parallax of the plane. Journal of the Optical Society of America, 66, 717-723. 
Longuet-Higgins, H., \& Prazdny, K. (1980). The interpretation of a moving retinal image. Proceedings of the Royal Society of London: Series $B, 208,385-397$.

Perrone, J. A. (1992). Model for the computation of self-motion in biological systems. Journal of the Optical Society of America A, 9, 177 194

Probst, T., KrafczyK, S., Brandt, T., \& Wist, E. R. (1984, August). Interaction between perceived self-motion and object-motion impairs vehicle guidance. Science, 225, 536-538.

REGAN, D., \& BeverLey, K. I. (1982, January). How do we avoid confounding the direction we are looking and the direction we are moving? Science, 215, 194-196.

Rieger J. H., \& Lawton, D. T. (1985). Processing differential image motion. Journal of the Optical Society of America A, 2, 354-359.

ROYDEN, C. S. (1994). Analysis of misperceived observer motion during simulated eye rotations. Vision Research, 34, 3215-3222.

Royden, C. S., Banks, M. S., \& Crowell, J. A. (1992). The perception of heading during eye movements. Nature, 360, 583-585.

Royden, C. S., Crowell, J. A., \& Banks, M. S. (1994). Estimating heading during eye movements. Vision Research, 34, 3197-3214.

ROYDEN, C. S., \& HILDRETH, E. C. (1996). Human heading judgments in the presence of moving objects. Perception \& Psychophysics, 58, 836-856.

Stoffregen, T. A. (1985). Flow structure versus retinal location in the optical control of stance. Journal of Experimental Psychology. Human Perception \& Performance, 11, 554-565.

VISHTON, P. M., \& CutTinG, J. E. (1995). Wayfinding, displacements, and mental maps: Velocity fields are not typically used to determine one's aimpoint. Journal of Experimental Psychology: Human Perception \& Performance, 21, 978-995.

voN HoLST, E. (1954). Relations between the central nervous system and the peripheral organs. British Journal of Animal Behaviour, 2, 89-94

WARREN, R. (1976). The perception of egomotion. Journal of Experimental Psychology: Human Perception \& Performance, 2, 448-456

WARREN, R. (1988). Visual perception in high-speed low-altitude flight. Aviation, Space, \& Environmental Medicine, 59, Al16-124.

WARREN, W. H. (1990). The perception-action coupling. In H. Bloch \& B. I. Bertenthal (Eds.), Sensory-motor organization in infancy and early childhood (pp. 23-37). Dordrecht: Kluwer.

WARREN, W. H., \& HANNON, D. J. (1988). Direction of self-motion is perceived from optical flow. Nature, 336, 162-163.

WARREN, W. H., \& HANNON, D. J. (1990). Eye movements and optical flow. Journal of the Optical Society of America A, 7, 160-169.

WARREN, W. H., \& KURTZ, K. J. (1992). The role of central and peripheral vision in perceiving the direction of self-motion. Perception \& Psychophysics, 51, 443-454.

Warren, W. H., Mestre, D., Blackwell, A., \& Morris, M. (1991). Perception of circular heading from optical flow. Journal of Experimental Psychology: Human Perception \& Performance, 17, 28-43.

Warren, W. H., Morris, M., \& Kalish, M. (1988). Perception of translational heading from optical flow. Journal of Experimental Psychology: Human Perception \& Performance, 14, 646-660.

Wertheim, A. H. (1990). Visual, vestibular, and oculomotor interactions in the perception of object motion during egomotion. In R. Warren \& A. H. Wertheim (Eds.), Perception \& control of self-motion (pp. 171-217). Hillsdale, NJ: Erlbaum.

\section{NOTES}

1. The problem of modeling locomotion perception in visual scenes is elaborated further when moving objects are added to the stationary objects in the visual scene (Probst, Krafczyk, Brandt, \& Wist, 1984; Royden \& Hildreth, 1996).

2. Though controlling locomotion also requires other competencies, moving observers must determine heading with some degree of accuracy if they are to regulate movement toward an object (or among objects) in the world. For this reason, findings from the considerable body of heading perception studies are considered in this paper in the discussions of both gaze-movement angle and extraretinal eye movement information.
3. This explanation must at some point consider that other classes of image transformation (such as divergence and deformation) occur in conjunction with rotation in the flow fields of moving observers; subjects do not perceive that they are moving forward along a curved path when they are presented with a display showing pure roll rotation.

4. The term camera will be used to denote a viewing configuration that includes a virtual eye point, a viewing direction, and a projection surface with a specified geometry.

5. Although the strongest gusts were in the forward-backward dimension, small gusts were imposed in the vertical dimension, and the subject's gaze was not restricted in Experiment 1. For this reason, the gaze-movement angle was always small but was not continuously zero in the baseline (horizon) condition. Note that if we apply the definition of gaze-movement angle rigorously here, moving backward while looking forward actually creates a gaze angle of $180^{\circ}$. Since forward motion and backward motion create similar but opposite image transformations (pure expansion and contraction, respectively) in the center of the image, we will consider the gaze-movement angles associated with forward movements and backward movements in the horizon condition to be near zero.

6 . When the hovering observer must contend with strong gusts in more than one dimension (e.g., up-down gusts in addition to forwardbackward gusts), however, mere gaze-heading discrimination may be insufficient to support optimal position control. Confusion regarding absolute heading could impede position control when similar image transformations (such as change in perceived slant) might be associated with a wide range of self-motion headings as opposed to a single heading. As discussed below, extraretinal eye movement information offers one of several possible mechanisms with which the observer could disambiguate such confusion.

7. This expression is obtained by integrating each component velocity function $V=A * \sin (\omega t)$ over one-half cycle, with $\omega=2 \pi F$.

8. As pointed out by Koenderink (1986), the optic flow field in this situation can be characterized by only four components, namely pure translation, expansion (div), rotation (curl), and deformation (def). (The def component specifies contraction in one dimension combined with expansion in the orthogonal dimension.) If we consider a forward-moving eye or camera pursuing a fixation location on the ground ahead, the translation component is canceled by the pursuit eye movement and there is negligible curl in the image. This leaves expansion and deformation as the only discernible transformations.

9. No eye position recording device was available to confirm the accuracy with which subjects were following instructions to pursue the fixation target. However, in the $-90^{\circ}$ condition, in which we might expect eye movement information to be most important, the optical distance between the central fixation target and the nearest texture element was approximately $20^{\circ}$. Since the background was solid between the texture elements, the subject would have had nothing to fixate that was not at least $20^{\circ}$ from the target even if he/she was lax about following instructions.

10. The result of this post hoc contrast (namely that at this camera orientation, the variable component of the position control error was greater for no-simulated-pursuit trials) is not immediately evident upon inspection of the figure. The contrast was based on a pooled error term. which is not directly comparable to the standard errors of the mean represented by the overlapping error bars in the figure.

11. This interpretation is consistent with the findings in the present study and with recent model explanations of the curved-path error in simulated-pursuit displays. It should be noted, however, that keeping the eyes stationary in the head while following a downward-veering forward path would be expected to bring about slip in the retinal flow field. Since this retinal slip was not displayed in the simulated-pursuit conditions, curved-path misperceptions, if they occurred in the present study, would not have corresponded exactly to the available visual information. It is possible that the information indicating slant and distance changes simply overpowered other information sources to bring about a curvilinear motion percept in the absence of information indicating ocular pursuit. As yet, however, the occurrence of curved-path misperceptions is only one possible account for the findings in Experiment 3

(Manuscript received October 8, 1993: revision accepted for publication November 1, 1997.) 